\title{
Deligne-Lusztig Induction for Invariant Functions on Finite Lie Algebras of Chevalley's Type
}

\author{
Emmanuel LETELLIER
}

Sophia University

(Communicated by K. Shinoda)

\begin{abstract}
Let $G$ be a connected reductive algebraic group defined over $\mathbf{F}_{q}$ with Lie algebra $\mathcal{G}$. We define a Deligne-Lusztig induction for the $\overline{\mathbf{Q}}_{\ell}$-valued functions on $\mathcal{G}\left(\mathbf{F}_{q}\right)$ which are invariant under the adjoint action of $G\left(\mathbf{F}_{q}\right)$ on $\mathcal{G}\left(\mathbf{F}_{q}\right)$, by making use of the "character formula" where the "two-variable Green functions" are defined via a $G$-equivariant homeomorphism $\mathcal{G}_{\text {nil }} \rightarrow G_{\text {uni }}$. We verify that it satisfies properties analogous to the group case like transitivity, the Mackey formula or the commutation with duality. The interest of a Deligne-Lusztig induction for invariant functions comes from a conjecture on a commutation formula with Fourier transforms which has no counterpart in the group case. In a forthcoming paper, this conjecture will be proved in almost all cases.
\end{abstract}

\section{Introduction}

Let $G$ be a connected reductive group over an algebraic closure $\mathbf{F}$ of the finite field $\mathbf{F}_{q}$ with $q$ elements and let $p$ be the characteristic of $\mathbf{F}$. Assume that $G$ is defined over $\mathbf{F}_{q}$ with associated Frobenius endomorphism $F$. Then the Lie algebra $\mathcal{G}$ of $G$ and the adjoint action of $G$ on $\mathcal{G}$ are also defined over $\mathbf{F}_{q}$. We still denote by $F$ the corresponding Frobenius endomorphism on $\mathcal{G}$. We then denote by $G^{F}$ (resp. $\mathcal{G}^{F}$ ) the set of the elements of $G$ (resp. $\mathcal{G}$ ) which are fixed by $F$. Let $\ell$ be a prime $\neq p$ and let $\overline{\mathbf{Q}}_{\ell}$ be an algebraic closure of the field $\mathbf{Q}_{\ell}$

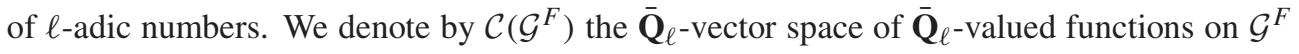
which are invariant under the adjoint action of $G^{F}$ on $\mathcal{G}^{F}$. Let $L$ be an $F$-stable Levi subgroup of a parabolic subgroup $P$ of $G$ and let $\mathcal{L}$ be the Lie algebra of $L$. If $P$ is $F$-stable, then we have the Lie algebra version of Harish-Chandra induction $\mathcal{C}\left(\mathcal{L}^{F}\right) \rightarrow \mathcal{C}\left(\mathcal{G}^{F}\right)$. The aim of this paper is to generalize this induction to the case where $P$ is not necessarily $F$-stable. In the group setting such a generalization, called Deligne-Lusztig induction, has been constructed in [DL76]. In [DM87][Lus86], we have a formula, called "character formula", which expresses the values of the Deligne-Lusztig induction of a class function $f$ on $L^{F}$ in terms of the values of $f$ and the values of some unipotently supported functions, called "two-variable Green functions" [DM87]. Our definition of Deligne-Lusztig induction in the Lie algebra setting uses the Lie algebra version of the character formula where the two-variable Green functions are transferred to the Lie algebras via a $G$-equivariant homeomorphism between the nilpotent 
subvariety $\mathcal{G}_{\text {nil }}$ of $\mathcal{G}$ and the unipotent subvariety $G_{\text {uni }}$ of $G$. The author was informed that Lusztig already knew this definition (unpublished). In a forthcoming paper, the author will use this definition of Deligne-Lusztig induction to prove for almost all $G$, a commutation formula between Fourier transforms and Deligne-Lusztig induction. Such a commutation formula was proved by Lehrer [Leh96] for Harish-Chandra induction. It will be also shown that this definition of Deligne-Lusztig induction does not depend on the choice of a $G$-equivariant homeomorphism $\mathcal{G}_{\text {nil }} \rightarrow G_{\text {uni }}$.

In this paper we start by recalling some well-known facts about the space $\mathcal{C}\left(\mathcal{G}^{F}\right)$ of $G^{F}$-invariant functions on $\mathcal{G}^{F}$. The second part will be devoted to the definition of DeligneLusztig induction; we will also verify elementary properties analogous to the group case like transitivity or the fact that it generalizes Harish-Chandra induction. In the fourth part, we will prove the Mackey formula (following Bonnafé's method) and its consequences, like the commutation with the duality map.

I wish to thank J. Michel and the referee for useful comments on this paper. This work has been supported by JSPS (Japanese Society for Promotion of Science).

\section{Preliminaries}

No assumption on $p$ is required unless otherwise specified.

Notation 0.0 .1 . Let $H$ be a linear algebraic group over $\mathbf{F}$. If $x \in H$, we denote by $x_{s}$ the semi-simple part of $x$ and by $x_{u}$ the unipotent part of $x$. We denote by $H^{o}$ the neutral component of $H$ and by $Z_{H}$ the center of $H$. If $x \in H$, the centralizer of $x$ in $H$ is denoted by $C_{H}(x)$; it will be more convenient to denote the neutral component of $C_{H}(x)$ by $C_{H}^{o}(x)$ rather than by $C_{H}(x)^{o}$. Let $\mathcal{H}=\operatorname{Lie}(H)$ be the Lie algebra of $H$, for $x \in \mathcal{H}$, we denote by $x_{s}$ the semi-simple part of $x$ and by $x_{n}$ the nilpotent part of $x$. The adjoint action of $H$ on $\mathcal{H}$ is denoted by $\mathrm{Ad}=\operatorname{Ad}_{H}$ and we put ad $=\operatorname{ad}_{\mathcal{H}}$ the differential of $\mathrm{Ad}$ at $1 \in H$. If $K$ is a subgroup of $H$, by " $K$-orbit of $\mathcal{H}$ ", we shall mean " $\operatorname{Ad}(K)$-orbit of $\mathcal{H}$ ". If $x \in \mathcal{H}$, then we denote by $C_{H}(x)$ the centralizer of $x$ in $H$ i.e. $C_{H}(x)=\{h \in H \mid \operatorname{Ad}(h) x=x\}$ and by $C_{\mathcal{H}}(x):=\{y \in \mathcal{H} \mid \operatorname{ad}(x)(y)=0\}$. If $x \in \mathcal{H}$ is semi-simple, we have $\operatorname{Lie}\left(C_{H}(x)\right)=C_{\mathcal{H}}(x)$ [Bor, 9.1].

Notation 0.0.2. Let now $G$ be a connected reductive algebraic group over $\mathbf{F}$ with Lie algebra $\mathcal{G}$. We assume that $G$ is defined over $\mathbf{F}_{q}$ and we denote by $F$ the corresponding Frobenius endomorphisms on $G$ and on $\mathcal{G}$. If $P$ is a parabolic subgroup of $G$, we will denote by $U_{P}$ the unipotent radical of $P$ and by $\mathcal{U}_{P}$ the Lie algebra of $U_{P}$. Recall that a Levi subgroup $L$ of a parabolic subgroup $P$ of $G$ is a closed subgroup $L$ of $P$ such that $P=L \ltimes U_{P}$. We will use the shorter expression "Levi subgroup of $G$ " instead of "Levi subgroup of a parabolic subgroup of $G$ ". We say that an $F$-stable Levi subgroup of $G$ is $G$-split if it is a Levi subgroup of an $F$-stable parabolic subgroup of $G$. The letter $T$ will denote a maximal torus of $G$. The dimension of $T$ is called the rank of $G$ and is denoted by $r k(G)$. We denote by $\Phi$ the root system of $G$ with respect to $T$. If $\alpha \in \Phi$, we denote by $\mathcal{G}_{\alpha}$ the one-dimensional 
F-vector space $\{x \in \mathcal{G} \mid \forall t \in T, \operatorname{Ad}(t) x=\alpha(t) x\}$ and by $U_{\alpha}$ the unique closed connected onedimensional unipotent subgroup of $G$ normalized by $T$ such that $\operatorname{Lie}\left(U_{\alpha}\right)=\mathcal{G}_{\alpha}$. Finally we denote by $G_{\text {uni }}$ the subvariety of unipotent elements of $G$ and by $\mathcal{G}_{\text {nil }}$ the subvariety of nilpotent elements of $\mathcal{G}$.

REMARK 0.0.3. We will have to consider the Lie algebra of the intersection of closed subgroups of $G$. This appears for instance in the Mackey formula. Let $M$ and $N$ be two closed subgroups of $G$, we always have

$(*) \operatorname{Lie}(M \cap N) \subset \operatorname{Lie}(M) \cap \operatorname{Lie}(N)$.

In general this inclusion is not an equality; it is an equality exactly when the quotient morphism $\pi: G \rightarrow G / N$ induces a separable morphism $M \rightarrow \pi(M)$, see [Bor, Proposition 6.12]. However if $M \cap N$ contains a maximal torus of $G$, then by [Bor, Proposition 13.20], the inclusion $(*)$ is an equality; note that [Bor, Corollary 13.21], which asserts that $(*)$ is an equality whenever $M$ and $N$ are normalized by a maximal torus of $G$, is not correct since the intersection of two subtori of a maximal torus of $G$ may have finite intersection while their Lie algebras have an intersection of strictly positive dimension. For instance, let $G=S L_{3}(\mathbf{F})$ and let $T$ be the maximal torus of $G$ consisting of diagonal matrices, then the set $Z_{G}$ is finite and it is the intersection of the two subtori $T_{\alpha}=\operatorname{Ker}(\alpha)$ and $T_{\beta}=\operatorname{Ker}(\beta)$ of $T$ where $\alpha: T \rightarrow \mathbf{F}$, $\left(t_{1}, t_{2}, t_{1}^{-1} t_{2}^{-1}\right) \mapsto t_{1} t_{2}^{-1}$ and $\beta: T \rightarrow \mathbf{F},\left(t_{1}, t_{2}, t_{1}^{-1} t_{2}^{-1}\right) \mapsto t_{2}^{2} t_{1}$. The intersection of the Lie algebras of $T_{\alpha}$ and $T_{\beta}$ is of dimension 0 unless $p=3$, in which case the intersection is of dimension 1 .

We will be interested only in the cases where the subgroups $M$ and $N$ in (*) are either equal to $L, L^{\prime}, U_{P}, U_{P^{\prime}}, P$ or $P^{\prime}$ where $P=L U_{P}$ and $P^{\prime}=L^{\prime} U_{P^{\prime}}$ are two Levi decompositions in $G$ such that $L \cap L^{\prime}$ contains a maximal torus $T$ of $G$. In any of these cases, the inclusion (*) is always an equality; the case where $M=U_{P}$ and $N$ is either $U_{P^{\prime}}, L^{\prime}$ or $P^{\prime}$ follows from the fact that the dimension of $M \cap N$ and the dimension of $\operatorname{Lie}(M) \cap \operatorname{Lie}(N)$ are respectively equal to the number of $\alpha \in \Phi$ such that $U_{\alpha} \subset M \cap N$ and the number of $\alpha \in \Phi$ such that $\mathcal{G}_{\alpha} \subset \operatorname{Lie}(M) \cap \operatorname{Lie}(N)$.

\section{The space of $G^{F}$-invariant functions on $\mathcal{G}^{F}$}

We mostly recall here the parts of [Leh96] which will be used in this paper.

Notation 1.0.4. Let $H$ be an $F$-stable closed subgroup of $G$ with Lie algebra $\mathcal{H}$. For any $x \in \mathcal{H}^{F}$, we denote by $\gamma_{x}^{H} \in \mathcal{C}\left(\mathcal{H}^{F}\right)$ the function which takes the value $\left|C_{H}(x)^{F}\right|$ on the $H^{F}$-orbit of $x$ and the value 0 elswhere. We denote by $\eta_{o}^{\mathcal{H}} \in \mathcal{C}\left(\mathcal{H}^{F}\right)$ the function which takes the value 1 on the set of nilpotent elements of $\mathcal{H}^{F}$ and the value 0 elsewhere.

NotATION 1.0.5. Throughout this paper, we choose once for all an automorphism $\overline{\mathbf{Q}}_{\ell} \rightarrow \overline{\mathbf{Q}}_{\ell}, x \mapsto \bar{x}$ such that $\bar{\zeta}=\zeta^{-1}$ for any root of unity $\zeta$ of $\overline{\mathbf{Q}}_{\ell}$. 
Definition 1.0.6. Let $H$ be an $F$-stable closed subgroup of $G$ with Lie algebra $\mathcal{H}$. For $f, g \in \mathcal{C}\left(\mathcal{H}^{F}\right)$, define the non-degenerate bilinear form $(,)_{\mathcal{H}^{F}}$ by,

$$
(f, g)_{\mathcal{H}^{F}}=\left|H^{F}\right|^{-1} \sum_{x \in \mathcal{H}^{F}} f(x) \overline{g(x)} .
$$

Note that for $x \in \mathcal{H}^{F}$ and $f \in \mathcal{C}\left(\mathcal{H}^{F}\right)$, we have $\left(f, \gamma_{x}^{H}\right)_{\mathcal{H}^{F}}=f(x)$ and $\left(\gamma_{x}^{H}, f\right)_{\mathcal{H}^{F}}=$ $\overline{f(x)}$.

Definition 1.0.7. Let $P$ be an $F$-stable parabolic subgroup of $G$ and $L$ be an $F$ stable Levi subgroup of $P$. Let $\mathcal{P}=\mathcal{L} \oplus \mathcal{U}_{P}$ be the Lie algebra decomposition corresponding to $P=L U_{P}$ and let $\pi_{\mathcal{P}}: \mathcal{P} \rightarrow \mathcal{L}$ be the canonical projection.

(i) The Harish-Chandra restriction ${ }^{*} \mathcal{R}_{\mathcal{L} \subset \mathcal{P}}^{\mathcal{G}}: \mathcal{C}\left(\mathcal{G}^{F}\right) \rightarrow \mathcal{C}\left(\mathcal{L}^{F}\right)$ is defined by the following formula

$$
{ }^{*} \mathcal{R}_{\mathcal{L} \subset \mathcal{P}}^{\mathcal{G}}(f)(x)=\left|U_{P}^{F}\right|^{-1} \sum_{y \in \mathcal{U}_{P}^{F}} f(x+y) .
$$

(ii) The Harish-Chandra induction $\mathcal{R}_{\mathcal{L} \subset \mathcal{P}}^{\mathcal{G}}: \mathcal{C}\left(\mathcal{L}^{F}\right) \rightarrow \mathcal{C}\left(\mathcal{G}^{F}\right)$ is defined by

$$
\mathcal{R}_{\mathcal{L} \subset \mathcal{P}}^{\mathcal{G}}(f)(x)=\left|P^{F}\right|^{-1} \sum_{\left\{g \in G^{F} \mid \operatorname{Ad}(g) x \in \mathcal{P}^{F}\right\}} f\left(\pi_{\mathcal{P}}(\operatorname{Ad}(g) x)\right) .
$$

We have the following proposition (see [Leh96]).

PROPOSITION 1.0.8. The maps ${ }^{*} \mathcal{R}_{\mathcal{L} \subset \mathcal{P}}^{\mathcal{G}}$ and $\mathcal{R}_{\mathcal{L} \subset \mathcal{P}}^{\mathcal{G}}$ are adjoint with respect to the forms $(,)_{\mathcal{G}^{F}}$ and $(,)_{\mathcal{L}^{F}}$. Moreover they are independent of $P$.

Notation 1.0.9. Since the map $\mathcal{R}_{\mathcal{L} \subset \mathcal{P}}^{\mathcal{G}}$ is independent of $P$, we write $\mathcal{R}_{\mathcal{L}}^{\mathcal{G}}$ instead of $\mathcal{R}_{\mathcal{L} \subset \mathcal{P}}^{\mathcal{G}}$

1.0.10. We define (following Kawanaka [Kaw82] in the Lie algebra case and Lusztig, Curtis and Alvis in the group case) the "duality map" $\mathcal{D}_{\mathcal{G}}: \mathcal{C}\left(\mathcal{G}^{F}\right) \rightarrow \mathcal{C}\left(\mathcal{G}^{F}\right)$. For any connected reductive group $H$ defined over $\mathbf{F}_{q}$, we denote by $r(H)$ the semi-simple $\mathbf{F}_{q}$-rank of $H$, i.e. the $\mathbf{F}_{q}$-rank of $H / Z_{H}^{o}$.

Definition 1.0.11. Let $B$ be an $F$-stable Borel subgroup of $G$. For $f \in \mathcal{C}\left(\mathcal{G}^{F}\right)$, we define $\mathcal{D}_{\mathcal{G}}(f)$ by

$$
\mathcal{D}_{\mathcal{G}}(f)=\sum_{P \supset B}(-1)^{r(P)} \mathcal{R}_{\mathcal{L}_{P}}^{\mathcal{G}} \circ{ }^{*} \mathcal{R}_{\mathcal{L}_{P}}^{\mathcal{G}}(f)
$$

where the summation is over the set of the $F$-stable parabolic subgroups $P$ of $G$ containing $B$ and where $\mathcal{L}_{P}$ denotes the Lie algebra of an arbitrarily chosen $F$-stable Levi subgroup of $P$. 
It is known that the map $\mathcal{D}_{\mathcal{G}}$ does not depend on the $F$-stable Borel subgroup $B$ and on the choice of the $\mathcal{L}_{P}$.

PROPOSITION 1.0.12. [Kaw82] We have the following assertions,

(i) The duality map $\mathcal{D}_{\mathcal{G}}$ is an isometry with respect to the form $(,)_{\mathcal{G}^{F}}$.

(ii) $\mathcal{D}_{\mathcal{G}}$ is an involution, i.e. $\mathcal{D}_{\mathcal{G}} \circ \mathcal{D}_{\mathcal{G}}=I d_{\mathcal{C}\left(\mathcal{G}^{F}\right)}$.

Proposition 1.0.13. [Leh96, Proposition 3.15] Let L be an F-stable G-split Levi subgroup of $G$ and let $\mathcal{L}=\operatorname{Lie}(L)$. Then

$$
\mathcal{D}_{\mathcal{G}} \circ \mathcal{R}_{\mathcal{L}}^{\mathcal{G}}=\mathcal{R}_{\mathcal{L}}^{\mathcal{G}} \circ \mathcal{D}_{\mathcal{L}}
$$

\section{Deligne-Lusztig induction: definition and basic properties}

2.1. Deligne-Lusztig induction for class functions. If $X$ is a variety over $\mathbf{F}$, then we denote by $H_{c}^{i}\left(X, \overline{\mathbf{Q}}_{\ell}\right)$ the $i$-th group of $\ell$-adic cohomology with compact support as in [Del77]. All what we need to know (in this paper) about these groups can be found in [DM91, Chapter 10].

2.1.1. Let $L$ be an $F$-stable Levi subgroup of $G$, let $P=L U_{P}$ be a Levi decomposition of a (possibly non $F$-stable) parabolic subgroup $P$ of $G$ and let $\mathcal{P}=\mathcal{L} \oplus \mathcal{U}_{P}$ be the corresponding Lie algebra decomposition. We denote by $\mathcal{L}_{G}$ the Lang map $G \rightarrow G, x \mapsto$ $x^{-1} F(x)$. The variety $\mathcal{L}_{G}^{-1}\left(U_{P}\right)$ is endowed with an action of $G^{F}$ on the left and with an action of $L^{F}$ on the right. By [DM91, Proposition 10.2], these actions induce actions on the cohomology and so make $H_{c}^{i}\left(\mathcal{L}_{G}^{-1}\left(U_{P}\right), \overline{\mathbf{Q}}_{\ell}\right)$ into a $G^{F}$-module- $L^{F}$. The virtual $\overline{\mathbf{Q}}_{\ell^{-}}$vector space $H_{c}^{*}\left(\mathcal{L}_{G}^{-1}\left(U_{P}\right)\right):=\sum_{i}(-1)^{i} H_{c}^{i}\left(\mathcal{L}_{G}^{-1}\left(U_{P}\right), \overline{\mathbf{Q}}_{\ell}\right)$ is thus a virtual $G^{F}$-module- $L^{F}$.

Notation 2.1.2. If $(g, l) \in G^{F} \times L^{F}$, define $S_{L \subset P}^{G}(g, l):=\operatorname{Trace}\left(\left(g, l^{-1}\right) \mid\right.$ $\left.H_{c}^{*}\left(\mathcal{L}_{G}^{-1}\left(U_{P}\right)\right)\right)$.

To each $L^{F}$-module $M$, corresponds thus a virtual $G^{F}$-module $R_{L \subset P}^{G}(M):=$

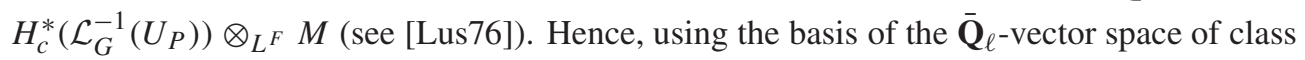
functions on $L^{F}$ formed by the irreducible characters of $L^{F}$, the map $R_{L \subset P}^{G}$ gives rise to a natural $\overline{\mathbf{Q}}_{\ell}$-linear map, so-called Deligne-Lusztig induction and still denoted by $R_{L \subset P}^{G}$, from the $\overline{\mathbf{Q}}_{\ell^{-v}}$-vector space of class functions on $L^{F}$ onto the $\overline{\mathbf{Q}}_{\ell^{-}}$vector space of class functions on $G^{F}$. More precisely if $f$ is a class function on $L^{F}$, the class function $R_{L \subset P}^{G}(f)$ on $G^{F}$ is given by the following formula:

2.1.3. $R_{L \subset P}^{G}(f)(g)=\left|L^{F}\right|^{-1} \sum_{h \in L^{F}} S_{L \subset P}^{G}(g, h) f(h) \quad$ for any $g \in G^{F}$.

REMARK 2.1.4. It is conjectured and proved for large enough values of $q$ that $R_{L \subset P}^{G}$ is independent of the parabolic subgroup $P$ having $L$ as a Levi subgroup (see section 3 for more details). 
We now define the two-variable Green functions; they appear naturally in the computation of the values of the Deligne-Lusztig induction of class functions (see 2.1.6 below).

Definition 2.1.5. The function $Q_{L \subset P}^{G}: G^{F} \times L^{F} \rightarrow \overline{\mathbf{Q}}_{\ell}$ defined by

$$
Q_{L \subset P}^{G}(u, v)= \begin{cases}\left|L^{F}\right|^{-1} \operatorname{Trace}\left(\left(u, v^{-1}\right) \mid H_{c}^{*}\left(\mathcal{L}_{G}^{-1}\left(U_{P}\right)\right)\right) & \text { if }(u, v) \in G_{u n i}^{F} \times L_{u n i}^{F}, \\ 0 & \text { otherwise }\end{cases}
$$

is called a two-variable Green function.

In the case where $L$ is a maximal torus of $G$, the two-variable Green functions become one-variable functions and are the ordinary Green functions introduced for any reductive groups by Deligne-Lusztig [DL76]. In the case of $G=G L_{n}(\mathbf{F})$, they were first introduced by Green [Gre55].

The following formula [DM91, 12.2][DM87][Lus86], the so-called character formula for $R_{L \subset P}^{G}$, expresses the values of the functions $R_{L \subset P}^{G}(f)$, where $f$ is a class function on $L^{F}$, in terms of the values of $f$ and in terms of the values of some two-variable Green functions:

2.1.6. For any $x \in G^{F}$,

$$
\begin{aligned}
& R_{L \subset P}^{G}(f)(x)= \\
& \quad\left|L^{F}\right|^{-1}\left|C_{G}^{o}\left(x_{s}\right)^{F}\right|^{-1} \sum_{\left\{h \in G^{F} \mid x_{s} \in \in^{h} L\right\}}\left|C_{h_{L}}^{o}\left(x_{s}\right)^{F}\right| \sum_{v \in\left(C_{h_{L}}^{o}\left(x_{s}\right)_{u n i}\right)^{F}} Q_{C_{h_{L}}^{o}\left(x_{s}\right)}^{C_{(}^{o}\left(x_{s}\right)}\left(x_{u}, v\right)^{h} f\left(x_{s} v\right)
\end{aligned}
$$

where ${ }^{h} L:=h L h^{-1}$ and ${ }^{h} f(y):=f\left(h^{-1} y h\right)$.

To simplify the notation, we usually omit the parabolic subgroup ${ }^{h} P \cap C_{G}^{o}\left(x_{s}\right)$ from the notation $Q_{C_{h_{L}}^{o}\left(x_{s}\right)}^{C_{0}^{o}\left(x_{s}\right)}$.

2.2. Deligne-Lusztig induction for invariant functions. In the Lie algebra setting, we define the Deligne-Lusztig induction using the Lie algebra version of the character formula where the two-variable Green functions are transfered to the Lie algebra by means of a $G$ equivariant homeomorphism $\mathcal{G}_{n i l} \rightarrow G_{u n i}$, where $G$ acts by conjugacy on $G_{u n i}$ and by the adjoint action on $\mathcal{G}_{\text {nil }}$.

Assumption 2.2.1. From now we assume that $p$ is $\operatorname{good}$ for $G$ so that there exists a $G$-equivariant homeomorphism $\phi: \mathcal{G}_{n i l} \rightarrow G_{\text {uni }}$ defined over $\mathbf{F}_{q}$ [Spr69].

Lemma 2.2.2. [Bon02, Lemma 3.2] For any Levi decomposition $P=L U_{P}$ in $G$ with corresponding Lie algebra decomposition $\mathcal{P}=\mathcal{L} \oplus \mathcal{U}_{P}$, we have:

(i) $\phi\left(\mathcal{L}_{\text {nil }}\right)=L_{\text {uni }}$,

(ii) for any $x \in \mathcal{L}_{n i l}, \phi\left(x+\mathcal{U}_{P}\right)=\phi(x) U_{P}$. 
Definition 2.2.3. With the notation of 2.1.1, the two-variable Green function $\mathcal{Q}_{\mathcal{L} \subset \mathcal{P}}^{\mathcal{G}}: \mathcal{G}^{F} \times \mathcal{L}^{F} \rightarrow \mathbf{Z}$ is defined by

$$
\mathcal{Q}_{\mathcal{L} \subset \mathcal{P}}^{\mathcal{G}}(u, v)= \begin{cases}\left|L^{F}\right|^{-1} \operatorname{Trace}\left(\left(\phi(u), \phi(v)^{-1}\right) \mid H_{c}^{*}\left(\mathcal{L}_{G}^{-1}\left(U_{P}\right)\right)\right) & \text { if }(u, v) \in \mathcal{G}_{\text {nil }}^{F} \times \mathcal{L}_{\text {nil }}^{F}, \\ 0 & \text { otherwise }\end{cases}
$$

REMARK 2.2.4. Assume that $\phi$ is the exponential map (which is well-defined if $p>$ $3\left(h_{o}^{G}-1\right)$ where $h_{o}^{G}$ is the Coxeter number of $\left.G\right)$. Let $T$ be an $F$-stable maximal torus of $G$ contained in a (possibly non $F$-stable) Borel subgroup $B$ of $G$. Assume that $\sigma \in \mathcal{T}^{F}$ satisfies $C_{G}^{o}(\sigma)=T$ and let $\mathcal{B}=\mathcal{T} \oplus \mathcal{U}_{B}$ be the Lie algebra decomposition corresponding to $B=$ $T U_{B}$. By a result of Kazhdan-Springer [Kaz77][Spr76], for any non-trivial additive character $\Psi: \mathbf{F}_{q} \rightarrow \overline{\mathbf{Q}}_{\ell}^{\times}$, any non-degenerate, symmetric, $G$-invariant bilinear form $\langle\rangle:, \mathcal{G} \times \mathcal{G} \rightarrow \mathbf{F}$ defined over $\mathbf{F}_{q}$, we have, for any $u \in \mathcal{G}_{\text {nil }}^{F}$ :

$$
\mathcal{Q}_{\mathcal{T} \subset \mathcal{B}}^{\mathcal{G}}(u, 0)=\varepsilon_{G} \varepsilon_{T} q^{\frac{|\Phi|}{2}} \sum_{x \in \mathcal{O}_{\sigma}^{G}} \Psi(\langle x, u\rangle)
$$

where $\varepsilon_{G}=(-1)^{\mathbf{F}_{q}-\operatorname{rank}(G)}$ and where $\mathcal{O}_{\sigma}^{G^{F}}$ denotes the $G^{F}$-orbit of $\sigma$.

Definition 2.2.5. Let $L$ be an $F$-stable Levi subgroup of $G$ and let $P=L U_{P}$ be a Levi decomposition of $P$ with corresponding Lie algebra decomposition $\mathcal{P}=\mathcal{L} \oplus \mathcal{U}_{P}$.

(i) Let $f \in \mathcal{C}\left(\mathcal{L}^{F}\right)$, then the Deligne-Lusztig induction $\mathcal{R}_{\mathcal{L} \subset \mathcal{P}}^{\mathcal{G}}(f) \in \mathcal{C}\left(\mathcal{G}^{F}\right)$ of $f$ is defined by

$$
\begin{aligned}
& \mathcal{R}_{\mathcal{L} \subset \mathcal{P}}^{\mathcal{G}}(f)\left(x_{s}+x_{n}\right) \\
& \quad=\left|L^{F}\right|^{-1}\left|C_{G}^{o}\left(x_{s}\right)^{F}\right|^{-1} \sum_{\left\{h \in G^{F} \mid x_{s} \in \epsilon^{h} \mathcal{L}\right\}}\left|C_{h_{L}}^{o}\left(x_{s}\right)^{F}\right| \sum_{v \in C_{h_{\mathcal{L}}}\left(x_{s}\right)_{n i l}^{F}} \mathcal{Q}_{C_{h_{\mathcal{L}}}\left(x_{s}\right)}^{C_{\mathcal{G}}\left(x_{s}\right)}\left(x_{n}, v\right) \operatorname{Ad}_{h}(f)\left(x_{s}+v\right)
\end{aligned}
$$

where for any $g \in G^{F},{ }^{g} L:=g L g^{-1},{ }^{g} \mathcal{L}=\operatorname{Ad}(g) \mathcal{L}$ and $\operatorname{Ad}_{g}: \mathcal{C}\left(\mathcal{L}^{F}\right) \rightarrow \mathcal{C}\left(\operatorname{Ad}(g) \mathcal{L}^{F}\right)$ is given by, $\operatorname{Ad}_{g}(f)(x)=f\left(\operatorname{Ad}\left(g^{-1}\right) x\right)$.

(ii) Let $g \in \mathcal{C}\left(\mathcal{G}^{F}\right)$, then the Deligne-Lusztig restriction ${ }^{*} \mathcal{R}_{\mathcal{L} \subset \mathcal{P}}^{\mathcal{G}}(g) \in \mathcal{C}\left(\mathcal{L}^{F}\right)$ of $g$ is defined by

$$
{ }^{*} \mathcal{R}_{\mathcal{L} \subset \mathcal{P}}^{\mathcal{G}}(g)\left(x_{s}+x_{n}\right)=\left|C_{L}^{o}\left(x_{s}\right)^{F}\right|\left|C_{G}^{o}\left(x_{s}\right)^{F}\right|^{-1} \sum_{u \in C_{\mathcal{G}}\left(x_{s}\right)_{n i l}^{F}} \mathcal{Q}_{C_{\mathcal{L}}\left(x_{s}\right)}^{C_{\mathcal{G}}\left(x_{s}\right)}\left(u, x_{n}\right) g\left(x_{s}+u\right) .
$$

The group version of 2.2.5(ii) is due to Digne-Michel [DM87].

REMARK 2.2.6. Since $p$ is good for $G$, the connected component of the centralizer in $G$ of a semi-simple element of $\mathcal{G}$ is a Levi subgroup of $G$. Indeed, if $\mathcal{T}$ is the Lie algebra of the maximal torus $T$ of $G$, then for $x \in \mathcal{T}$, the set $\{\alpha \in \Phi \mid d \alpha(x)=0\}$, where $d \alpha: \mathcal{T} \rightarrow \mathbf{F}$ is the differential of $\alpha$ at 1, is a $\mathbf{Q}$-closed root subsystem of $\Phi$ [Slo80, 3.14]. Hence, with the notation of 2.2.5, the map $\phi$ induces a well-defined map $C_{\mathcal{G}}\left(x_{s}\right)_{n i l} \rightarrow C_{G}^{o}\left(x_{s}\right)_{u n i}$. 
REMARK 2.2.7. The notation $\mathcal{R}_{\mathcal{L} \subset \mathcal{P}}^{\mathcal{G}}$ is used both for Deligne-Lusztig induction and Harish-Chandra induction; this is justified by 2.3.7.

OPEN PRoblem 2.2.8. Define $\mathcal{R}_{\mathcal{L} \subset \mathcal{P}}^{\mathcal{G}}$ using $\ell$-adic cohomology but without using any $G$-equivariant homeomorphism $\mathcal{G}_{\text {nil }} \rightarrow G_{\text {uni }}$.

REMARK 2.2.9. It follows easily from the formulae of 2.2 .5 that

(i) for any $f \in \mathcal{C}\left(\mathcal{L}^{F}\right)$, we have

$$
\mathcal{R}_{\mathcal{L} \subset \mathcal{P}}^{\mathcal{G}}\left(f . \eta_{o}^{\mathcal{L}}\right)=\mathcal{R}_{\mathcal{L} \subset \mathcal{P}}^{\mathcal{G}}(f) . \eta_{o}^{\mathcal{G}},
$$

(ii) for any $g \in \mathcal{C}\left(\mathcal{G}^{F}\right)$, we have

$$
{ }^{*} \mathcal{R}_{\mathcal{L} \subset \mathcal{P}}^{\mathcal{G}}\left(g . \eta_{o}^{\mathcal{G}}\right)={ }^{*} \mathcal{R}_{\mathcal{L} \subset \mathcal{P}}^{\mathcal{G}}(g) . \eta_{o}^{\mathcal{L}} .
$$

\subsection{Basic properties of $\mathcal{R}_{\mathcal{L} \subset \mathcal{P}}^{\mathcal{G}}$}

In this section, we prove the transitivity of the $\mathcal{R}_{\mathcal{L} \subset \mathcal{P}}^{\mathcal{G}}$. We also verify that $\mathcal{R}_{\mathcal{L} \subset \mathcal{P}}^{\mathcal{G}}$ coincides with Harish-Chandra induction if $P$ is $F$-stable, and that ${ }^{*} \mathcal{R}_{\mathcal{L} \subset \mathcal{P}}^{\mathcal{G}}$ and $\mathcal{R}_{\mathcal{L} \subset \mathcal{P}}^{\mathcal{G}}$ are adjoint with respect to $(,)_{\mathcal{L}^{F}}$ and $(,)_{\mathcal{G}^{F}}$. Note that the group version of these properties are proved from the general properties of generalized induction associated to a bi-module [DM91, Chapters 4, 11], and so it is not possible to adapt these proofs to our Lie algebra version of Deligne-Lusztig induction; we will thus come down to problems on two-variable Green functions.

As it can be seen from 2.1.3, the function $S_{L \subset P}^{G}: G^{F} \times L^{F} \rightarrow \overline{\mathbf{Q}}_{\ell}$ plays a fundamental role in Deligne-Lusztig's theory. We would like to have such a function in the Lie algebra case; this is possible thanks to [DM91, Lemma 12.3] which gives an expression of $S_{L \subset P}^{G}(g, l)$ (where $g \in G^{F}, l \in L^{F}$ ) in terms of the values of some two-variable Green functions. More precisely the function $S_{\mathcal{L} \subset \mathcal{P}}^{\mathcal{G}}: \mathcal{G}^{F} \times \mathcal{L}^{F} \rightarrow \overline{\mathbf{Q}}_{\ell}$ we are looking for is defined as follows:

Definition 2.3.1. For $x \in \mathcal{G}^{F}, y \in \mathcal{L}^{F}$, we define $S_{\mathcal{L} \subset \mathcal{P}}^{\mathcal{G}}(x, y)$ by

$$
S_{\mathcal{L} \subset \mathcal{P}}^{\mathcal{G}}(x, y)=\sum_{\left\{h \in G^{F} \mid \operatorname{Ad}(h) y_{s}=x_{s}\right\}}\left|C_{L}^{o}\left(y_{s}\right)^{F}\right|\left|C_{G}^{o}\left(y_{s}\right)^{F}\right|^{-1} \mathcal{Q}_{C_{\mathcal{L}}\left(y_{s}\right)}^{C_{\mathcal{G}}\left(y_{s}\right)}\left(\operatorname{Ad}\left(h^{-1}\right) x_{n}, y_{n}\right) .
$$

REMARK 2.3.2. Note that $S_{\mathcal{L} \subset \mathcal{P}}^{\mathcal{G}}(x, y)=\left|L^{F}\right| \mathcal{Q}_{\mathcal{L} \subset \mathcal{P}}^{\mathcal{G}}(x, y)$ for any $(x, y) \in \mathcal{G}_{\text {nil }}^{F} \times$ $\mathcal{L}_{\text {nil }}^{F}$.

The following lemma is the Lie algebra version of 2.1.3:

Lemma 2.3.3. Let $f \in \mathcal{C}\left(\mathcal{G}^{F}\right), g \in \mathcal{C}\left(\mathcal{L}^{F}\right)$, we have

$$
\text { (1) } \mathcal{R}_{\mathcal{L} \subset \mathcal{P}}^{\mathcal{G}}(g)(x)=\left|L^{F}\right|^{-1} \sum_{y \in \mathcal{L}^{F}} S_{\mathcal{L} \subset \mathcal{P}}^{\mathcal{G}}(x, y) g(y),
$$


(2) ${ }^{*} \mathcal{R}_{\mathcal{L} \subset \mathcal{P}}^{\mathcal{G}}(f)(y)=\left|G^{F}\right|^{-1} \sum_{x \in \mathcal{G}^{F}} S_{\mathcal{L} \subset \mathcal{P}}^{\mathcal{G}}(x, y) f(x)$.

ProOF. We first prove (2).

$$
\begin{aligned}
\left|G^{F}\right|^{-1} \sum_{x \in \mathcal{G}^{F}} S_{\mathcal{L} \subset \mathcal{P}}^{\mathcal{G}}(x, y) f(x) \\
=\left|G^{F}\right|^{-1}\left|C_{L}^{o}\left(y_{s}\right)^{F}\right|\left|C_{G}^{o}\left(y_{s}\right)^{F}\right|^{-1} \sum_{x \in \mathcal{G}^{F}} \sum_{\left\{h \in G^{F} \mid \operatorname{Ad}(h) y_{s}=x_{s}\right\}} \mathcal{Q}_{C_{\mathcal{L}}\left(y_{s}\right)}^{C_{\mathcal{G}}\left(y_{s}\right)}\left(\operatorname{Ad}\left(h^{-1}\right) x_{n}, y_{n}\right) f(x) \\
=\left|G^{F}\right|^{-1}\left|C_{L}^{o}\left(y_{s}\right)^{F}\right|\left|C_{G}^{o}\left(y_{s}\right)^{F}\right|^{-1} \\
\quad \times \sum_{h \in G^{F}} \sum_{x_{n} \in C_{\mathcal{G}}\left(\operatorname{Ad}(h) y_{s}\right)_{n i l}^{F}} \mathcal{Q}_{C_{\mathcal{L}}\left(y_{s}\right)}^{C_{\mathcal{G}}\left(y_{s}\right)}\left(\operatorname{Ad}\left(h^{-1}\right) x_{n}, y_{n}\right) f\left(\operatorname{Ad}(h) y_{s}+x_{n}\right) \\
=\left|G^{F}\right|^{-1}\left|C_{L}^{o}\left(y_{s}\right)^{F}\right|\left|C_{G}^{o}\left(y_{s}\right)^{F}\right|^{-1} \\
\quad \times \sum_{h \in G^{F}} \sum_{x_{n} \in C_{\mathcal{G}}\left(y_{s}\right)_{n i l}^{F}} \mathcal{Q}_{C_{\mathcal{L}}\left(y_{s}\right)}^{C_{\mathcal{G}}\left(y_{s}\right)}\left(x_{n}, y_{n}\right) f\left(y_{s}+x_{n}\right)={ }^{*} \mathcal{R}_{L \subset P}^{G}(f)(y)
\end{aligned}
$$

Using the $G$-equivariance of $\phi$, it is straightforward to see that,

$$
S_{\mathcal{L} \subset \mathcal{P}}^{\mathcal{G}}(x, y)=\sum_{h \in G^{F} \mid \operatorname{Ad}(h) y_{s}=x_{s}}\left|C_{h_{L}}^{o}\left(x_{s}\right)^{F}\right|\left|C_{G}^{o}\left(x_{s}\right)^{F}\right|^{-1} \mathcal{Q}_{C_{h_{\mathcal{L}}}\left(x_{s}\right)}^{C_{\mathcal{G}}\left(x_{s}\right)}\left(x_{n}, \operatorname{Ad}(h) y_{n}\right) .
$$

It is then not difficult to get (1).

PROPOSITION 2.3.4. The maps $\mathcal{R}_{\mathcal{L} \subset \mathcal{P}}^{\mathcal{G}}$ and ${ }^{*} \mathcal{R}_{\mathcal{L} \subset \mathcal{P}}^{\mathcal{G}}$ are adjoint with respect to the forms $(,)_{\mathcal{G}^{F}}$ and $(,)_{\mathcal{L}^{F}}$.

Proof. Let $g \in \mathcal{C}\left(\mathcal{L}^{F}\right)$ and $f \in \mathcal{C}\left(\mathcal{G}^{F}\right)$. We have

$$
\begin{aligned}
\left(f, \mathcal{R}_{L \subset P}^{G}(g)\right)_{\mathcal{G}^{F}} & =\left|G^{F}\right|^{-1} \sum_{x \in \mathcal{G}^{F}} f(x) \overline{\mathcal{R}_{\mathcal{L} \subset \mathcal{P}}^{\mathcal{G}}(g)(x)} \\
& =\left|L^{F}\right|^{-1}\left|G^{F}\right|^{-1} \sum_{x \in \mathcal{G}^{F}} \sum_{y \in \mathcal{L}^{F}} f(x) \overline{S_{\mathcal{L} \subset \mathcal{P}}^{\mathcal{G}}(x, y) g(y)} \quad \text { by 2.3.3(1) } \\
& =\left|L^{F}\right|^{-1}\left|G^{F}\right|^{-1} \sum_{y \in \mathcal{L}^{F}} \sum_{x \in \mathcal{G}^{F}} S_{\mathcal{L} \subset \mathcal{P}}^{\mathcal{G}}(x, y) f(x) \overline{g(y)}
\end{aligned}
$$

The last equality follows from the fact that $S_{\mathcal{L} \subset \mathcal{P}}^{\mathcal{G}}(x, y) \in \mathbf{Q}$. We thus get from 2.3 .3 (2) that $\left(f, \mathcal{R}_{\mathcal{L} \subset \mathcal{P}}^{\mathcal{G}}(g)\right)_{\mathcal{G}^{F}}=\left({ }^{*} \mathcal{R}_{\mathcal{L} \subset \mathcal{P}}^{\mathcal{G}}(f), g\right)_{\mathcal{L}^{F}}$.

We now prove the transitivity of Deligne-Lusztig induction, that is, if $M \subset L \subset G$ is an inclusion of $F$-stable Levi subgroups of $G$, we have $\mathcal{R}_{\mathcal{L} \subset \mathcal{P}}^{\mathcal{G}} \circ \mathcal{R}_{\mathcal{M} \subset \mathcal{L} \cap \mathcal{Q}}^{\mathcal{L}}=\mathcal{R}_{\mathcal{M} \subset \mathcal{Q}}^{\mathcal{G}}$ 
where $\mathcal{L}=\operatorname{Lie}(L)$ and $\mathcal{M}=\operatorname{Lie}(M)$ and where $\mathcal{P}=\operatorname{Lie}(P)$ and $\mathcal{Q}=\operatorname{Lie}(Q)$ with $P, Q$ two parabolic subgroups of $G$ having respectively $L$ and $M$ as Levi subgroup and such that $Q \subset P$. We start by proving a "transitivity formula" for two-variable Green functions:

LEMMA 2.3.5. With the above notation, for any $(x, z) \in \mathcal{G}_{\text {nil }}^{F} \times \mathcal{M}_{\text {nil }}^{F}$, we have

$$
\mathcal{Q}_{\mathcal{M} \subset \mathcal{Q}}^{\mathcal{G}}(x, z)=\sum_{v \in \mathcal{L}_{\text {nil }}^{F}} \mathcal{Q}_{\mathcal{L} \subset \mathcal{P}}^{\mathcal{G}}(x, v) \mathcal{Q}_{\mathcal{M} \subset \mathcal{L} \cap \mathcal{Q}}^{\mathcal{L}}(v, z)
$$

Proof. The lemma will follow from its group version. From the proof of [DM91, 11.5], we have

$$
S_{M \subset Q}^{G}(x, z)=\left|L^{F}\right|^{-1} \sum_{y \in L^{F}} S_{L \subset P}^{G}(x, y) S_{M \subset L \cap Q}^{G}(y, z)
$$

for any $(x, z) \in G_{u n i}^{F} \times M_{u n i}^{F}$. By [DM91, Lemma 12.3], we have $S_{L \subset P}^{G}(x, y)=0$ if $x_{s}$ and $y_{s}$ are not $G^{F}$-conjugate. Hence for any $(x, z) \in G_{u n i}^{F} \times M_{u n i}^{F}$, we deduce that

$$
S_{M \subset Q}^{G}(x, z)=\left|L^{F}\right|^{-1} \sum_{y \in L_{u n i}^{F}} S_{L \subset P}^{G}(x, y) S_{M \subset L \cap Q}^{G}(y, z) .
$$

It follows that

$$
Q_{M \subset Q}^{G}(x, z)=\sum_{y \in L_{u n i}^{F}} Q_{L \subset P}^{G}(x, y) Q_{M \subset L \cap Q}^{L}(y, z)
$$

for any $(x, z) \in G_{u n i}^{F} \times M_{u n i}^{F}$.

PROPOSITION 2.3.6. We have

$$
\mathcal{R}_{\mathcal{L} \subset \mathcal{P}}^{\mathcal{G}} \circ \mathcal{R}_{\mathcal{M} \subset \mathcal{L} \cap \mathcal{Q}}^{\mathcal{L}}=\mathcal{R}_{\mathcal{M} \subset \mathcal{Q}}^{\mathcal{G}}
$$

PROOF. Thanks to 2.3.3 (1), it is enough to prove the following statement: for any $x \in \mathcal{G}^{F}, z \in \mathcal{M}^{F}$, we have

$$
\left|L^{F}\right|^{-1} \sum_{y \in \mathcal{L}^{F}} S_{\mathcal{L} \subset \mathcal{P}}^{\mathcal{G}}(x, y) S_{\mathcal{M} \subset \mathcal{Q} \cap \mathcal{L}}^{\mathcal{L}}(y, z)=S_{\mathcal{M} \subset \mathcal{Q}}^{\mathcal{G}}(x, z) .
$$

Now a simple calculation shows that this statement reduces to 2.3.5.

We have the following proposition:

Proposition 2.3.7. If the parabolic subgroup $P$ is F-stable, then the DeligneLusztig induction $\mathcal{R}_{\mathcal{L} \subset \mathcal{P}}^{\mathcal{G}}$ coincides with Harish-Chandra induction. 
PROOF. From the adjunction property 2.3.4 it is equivalent to prove that DeligneLusztig restriction ${ }^{*} \mathcal{R}_{\mathcal{L} \subset \mathcal{P}}^{\mathcal{G}}$ coincides with Harish-Chandra restriction. Let $(x, y) \in \mathcal{G}^{F} \times \mathcal{L}^{F}$. We first compute the quantity $S_{\mathcal{L} \subset \mathcal{P}}^{\mathcal{G}}(x, y)$. Define $L_{y_{s}}:=C_{L}^{o}\left(y_{s}\right), G_{y_{s}}:=C_{G}^{o}\left(y_{s}\right)$ and $V_{y_{s}}:=G_{y_{s}} \cap U_{P}$. Let $\mathcal{L}_{y_{s}}=\operatorname{Lie}\left(L_{y_{s}}\right), \mathcal{G}_{y_{s}}=\operatorname{Lie}\left(G_{y_{s}}\right)$ and $\mathcal{V}_{y_{s}}=\operatorname{Lie}\left(V_{y_{s}}\right)$; then $L_{y_{s}} V_{y_{s}}$ is a Levi decomposition of the parabolic subgroup $P \cap G_{y_{s}}$ of $G_{y_{s}}$. We denote by $\mathcal{L}_{G_{y_{s}}}: G_{y_{s}} \rightarrow G_{y_{s}}$ the Lang map $x \mapsto x^{-1} F(x)$. Since $V_{y_{s}}$ is $F$-stable, by [DM91, p. 81], the bi-module $H_{c}^{*}\left(\mathcal{L}_{G_{y_{s}}}^{-1}\left(V_{y_{s}}\right)\right)$ is isomorphic as $G_{y_{s}}^{F}$-module- $L_{y_{s}}^{F}$ to $\overline{\mathbf{Q}}_{\ell}\left[G_{y_{s}}^{F} / V_{y_{s}}^{F}\right]$. Let $h \in G^{F}$ be such that $\operatorname{Ad}(h) y_{s}=x_{s}$. Then we have

$$
\begin{aligned}
\mathcal{Q}_{\mathcal{L}_{y_{s}}}^{\mathcal{G}_{y_{s}}}\left(\operatorname{Ad}\left(h^{-1}\right) x_{n}, y_{n}\right) & =\left|L_{y_{s}}^{F}\right|^{-1} \operatorname{Trace}\left(\left(h^{-1} \phi\left(x_{n}\right) h, \phi\left(y_{n}\right)^{-1}\right) \mid \overline{\mathbf{Q}}_{\ell}\left[G_{y_{s}}^{F} / V_{y_{s}}^{F}\right]\right) \\
& =\left|L_{y_{s}}^{F}\right|^{-1} \sharp\left\{z V_{y_{s}}^{F} \in\left(G_{y_{s}}^{F} / V_{y_{s}}^{F}\right) \mid(h z)^{-1} \phi\left(x_{n}\right) h z \in \phi\left(y_{n}\right) V_{y_{s}}^{F}\right\} .
\end{aligned}
$$

From the $G$-equivariance of $\phi$, we get that

$$
\begin{aligned}
& \mathcal{Q}_{\mathcal{L}_{y_{s}}}^{\mathcal{G}_{y_{s}}}\left(\operatorname{Ad}\left(h^{-1}\right) x_{n}, y_{n}\right)=\left|L_{y_{s}}^{F}\right|^{-1} \sharp\left\{z V_{y_{s}}^{F} \in\left(G_{y_{s}}^{F} / V_{y_{s}}^{F}\right) \mid \phi\left(\operatorname{Ad}\left((h z)^{-1}\right) x_{n}\right) \in \phi\left(y_{n}\right) V_{y_{s}}^{F}\right\} \\
& =\left|L_{y_{s}}^{F}\right|^{-1} \sharp\left\{z V_{y_{s}}^{F} \in\left(G_{y_{s}}^{F} / V_{y_{s}}^{F}\right) \mid \operatorname{Ad}\left((h z)^{-1}\right) x_{n} \in y_{n}+\mathcal{V}_{y_{s}}^{F}\right\} \quad \text { by } 2.2 .2 \\
& =\left|L_{y_{s}}^{F}\right|^{-1} \sharp\left\{z V_{y_{s}}^{F} \in\left(G_{y_{s}}^{F} / V_{y_{s}}^{F}\right) \mid \operatorname{Ad}\left((h z)^{-1}\right) x \in y+\mathcal{V}_{y_{s}}^{F}\right\} \text {. }
\end{aligned}
$$

We deduce that

$$
S_{\mathcal{L} \subset \mathcal{P}}^{\mathcal{G}}(x, y)=\left|G_{y_{s}}^{F}\right|^{-1} \sum_{\left\{h \in G^{F} \mid \operatorname{Ad}(h) y_{s}=x_{s}\right\}} \sharp\left\{z V_{y_{s}}^{F} \in\left(G_{y_{s}}^{F} / V_{y_{s}}^{F}\right) \mid \operatorname{Ad}\left((h z)^{-1}\right) x \in y+\mathcal{V}_{y_{s}}^{F}\right\} .
$$

Thus for any $f \in \mathcal{C}\left(\mathcal{G}^{F}\right)$, we have:

$$
\begin{aligned}
& { }^{*} \mathcal{R}_{\mathcal{L} \subset \mathcal{P}}^{\mathcal{G}}(f)(y)=\left|G^{F}\right|^{-1}\left|G_{y_{s}}^{F}\right|^{-1} \\
& \quad \times \sum_{x \in \mathcal{G}^{F}} \sum_{\left\{h \in G^{F} \mid \operatorname{Ad}(h) y_{s}=x_{s}\right\}} \sharp\left\{z V_{y_{s}}^{F} \in\left(G_{y_{s}}^{F} / V_{y_{s}}^{F}\right) \mid \operatorname{Ad}\left((h z)^{-1}\right) x \in y+\mathcal{V}_{y_{s}}^{F}\right\} f(x) .
\end{aligned}
$$

By interchanging the sums we get that

$$
\begin{aligned}
& { }^{*} \mathcal{R}_{\mathcal{L} \subset \mathcal{P}}^{\mathcal{G}}(f)(y)=\left|G^{F}\right|^{-1}\left|G_{y_{s}}^{F}\right|^{-1} \\
& \quad \times \sum_{h \in G^{F}} \sum_{\left\{x \in \mathcal{G}^{F} \mid x_{s}=\operatorname{Ad}(h) y_{s}\right\}} \sharp\left\{z V_{y_{s}}^{F} \in\left(G_{y_{s}}^{F} / V_{y_{s}}^{F}\right) \mid \operatorname{Ad}\left((h z)^{-1}\right) x \in y+\mathcal{V}_{y_{s}}^{F}\right\} f(x) .
\end{aligned}
$$

We deduce that,

$$
{ }^{*} \mathcal{R}_{\mathcal{L} \subset \mathcal{P}}^{\mathcal{G}}(f)(y)=\left|G^{F}\right|^{-1}\left|G_{y_{s}}^{F}\right|^{-1} \sum_{h \in G^{F}} \sum_{\substack{x \in \mathcal{G} F \\ x_{s}=\operatorname{Ad}(h) y_{s}}} \sum_{\substack{\left.z V_{y_{s}}^{F} \in\left(G_{y^{\prime}}^{F} / V_{y_{s}}^{F}\right) \\ \operatorname{Ad}(h z)^{-1}\right) x \in y+\mathcal{V}_{y_{s}}^{F}}} f(x) .
$$


By interchanging the second with the third sum, we get that

$$
{ }^{*} \mathcal{R}_{\mathcal{L} \subset \mathcal{P}}^{\mathcal{G}}(f)(y)=\left|G^{F}\right|^{-1}\left|G_{y_{s}}^{F}\right|^{-1} \sum_{h \in G^{F}} \sum_{\left\{z V_{y_{s}}^{F} \in\left(G_{y_{s}}^{F} / V_{y_{s}}^{F}\right)\right\}} \sum_{\substack{x \in \mathcal{G} F \\ x \in \operatorname{Ad}(h z)\left(y+\mathcal{V}_{y_{s}}^{F}\right)}} f(x) .
$$

Since the function $f$ is $G^{F}$-invariant, we deduce that

$$
{ }^{*} \mathcal{R}_{\mathcal{L} \subset \mathcal{P}}^{\mathcal{G}}(f)(y)=\left|G^{F}\right|^{-1}\left|G_{y_{s}}^{F}\right|^{-1} \sum_{h \in G^{F}} \sum_{\left\{z V_{y s}^{F} \in\left(G_{y s}^{F} / V_{y s}^{F}\right)\right\}} \sum_{v \in \mathcal{V}_{y s}^{F}} f(y+v) .
$$

Hence

$$
{ }^{*} \mathcal{R}_{\mathcal{L} \subset \mathcal{P}}^{\mathcal{G}}(f)(y)=\left|V_{y_{s}}^{F}\right|^{-1} \sum_{v \in \mathcal{V}_{y_{s}}^{F}} f(y+v)
$$

To complete the proof, we need the following proposition (which is the Lie algebra version of [DM91, Proposition 7.1]):

Proposition 2.3.8. With the above notation, let $h: U_{P}^{F} \times \mathcal{V}_{y_{s}}^{F} \rightarrow y+\mathcal{U}_{P}^{F}$ be the morphism given by $h(u, v)=A d(u)(y+v)$. Then $h$ is surjective and the cardinality of its fibers is equal to $\left|V_{y_{s}}^{F}\right|$.

ProOF OF 2.3.8. Since $y \in \mathcal{L}$, the map $h$ is well-defined. To prove the surjectivity of $h$ it is enough to prove that $|\operatorname{Im}(h)|=\left|U_{P}^{F}\right|$. Let $X=\operatorname{Im}(h)$ and $z \in X$. There exists $\delta \in U_{P}^{F}$ and $v \in \mathcal{V}_{y_{s}}^{F}$ such that $z=\operatorname{Ad}(\delta)(y+v)$. Now the map $h^{-1}(z) \rightarrow h^{-1}(y)$ which sends $(\gamma, w)$ onto $\left(\delta^{-1} \gamma, w-\operatorname{Ad}\left(\gamma^{-1} \delta\right) v\right)$ is a bijection whose inverse is given by $(a, x) \mapsto$ $\left(\delta a, x+\operatorname{Ad}\left(a^{-1}\right) v\right)$. Hence the fibers of the map $h: U_{P}^{F} \times \mathcal{V}_{y_{s}}^{F} \rightarrow X$ are all of same cardinality equal to $\left|h^{-1}(y)\right|$. We deduce that $|X|=\frac{\left|U_{P}^{F}\right|\left|\mathcal{V}_{y s}^{F}\right|}{\left|h^{-1}(y)\right|}$. Thus we need to prove that $\left|h^{-1}(y)\right|=\left|\mathcal{V}_{y_{s}}^{F}\right|$. Since $y \in \mathcal{L}_{y_{s}}$, we have $\operatorname{Ad}(u) y-y \in \mathcal{V}_{y_{s}}$ for any $u \in V_{y_{s}}$. We thus have an injective map $\psi: V_{y_{s}}^{F} \rightarrow h^{-1}(y)$ mapping $u$ onto $\left(u, \operatorname{Ad}\left(u^{-1}\right) y-y\right)$. It remains to prove the surjectivity of $\psi$. Let $(\delta, v) \in h^{-1}(y)$; we have $\operatorname{Ad}(\delta)(y+v)=y$. Since $y \in \mathcal{L}_{y_{s}}$, by [Leh96, 3.7], there exists $\zeta \in V_{y_{s}}$ such that $\operatorname{Ad}(\zeta) y_{s}=(y+v)_{s}$. We thus have $\operatorname{Ad}(\delta \zeta) y_{s}=y_{s}$ from which we deduce that $\delta \in G_{y_{s}} \cap U_{P}=V_{y_{s}}$ which proves the surjectivity of $\psi$ since $\psi(\delta)=(\delta, v)$.

From 2.3.8 and (1) we deduce that

$$
{ }^{*} \mathcal{R}_{\mathcal{L} \subset \mathcal{P}}^{\mathcal{G}}(f)(y)=\left|U_{P}^{F}\right|^{-1} \sum_{v \in \mathcal{U}_{P}^{F}} f(y+v) .
$$

Hence ${ }^{*} \mathcal{R}_{\mathcal{L} \subset \mathcal{P}}^{\mathcal{G}}$ coincides with Harish-Chandra restriction. 
Proposition 2.3.9. Let $L$ be an $F$-stable Levi subgroup of $G$ and $P$ be a parabolic subgroup of $G$ having $L$ as a Levi subgroup. Let $\mathcal{L}:=\operatorname{Lie}(L)$ and $\mathcal{P}:=\operatorname{Lie}(P)$. Let $x \in \mathcal{L}^{F}$ be such that $C_{G}^{o}\left(x_{s}\right) \subseteq L$, then $\mathcal{R}_{\mathcal{L} \subset \mathcal{P}}^{\mathcal{G}}\left(\gamma_{x}^{L}\right)=\gamma_{x}^{G}$

Proof. We compute the values of $\mathcal{R}_{\mathcal{L} \subset \mathcal{P}}^{\mathcal{G}}\left(\gamma_{x}^{L}\right)$. Let $y \in \mathcal{G}^{F}$, then

$$
\left(\mathcal{R}_{\mathcal{L} \subset \mathcal{P}}^{\mathcal{G}}\left(\gamma_{x}^{L}\right), \gamma_{y}^{G}\right)_{\mathcal{G}^{F}}=\mathcal{R}_{\mathcal{L} \subset \mathcal{P}}^{\mathcal{G}}\left(\gamma_{x}^{L}\right)(y)
$$

From 2.3.4 we have

$$
\left(\mathcal{R}_{\mathcal{L} \subset \mathcal{P}}^{\mathcal{G}}\left(\gamma_{x}^{L}\right), \gamma_{y}^{G}\right)_{\mathcal{G} F}=\left(\gamma_{x}^{L},{ }^{*} \mathcal{R}_{\mathcal{L} \subset \mathcal{P}}^{\mathcal{G}}\left(\gamma_{y}^{G}\right)\right)_{\mathcal{L}^{F}}
$$

Combining the above two equations we get that

$$
\mathcal{R}_{\mathcal{L} \subset \mathcal{P}}^{\mathcal{G}}\left(\gamma_{x}^{L}\right)(y)=\overline{* \mathcal{R}_{\mathcal{L} \subset \mathcal{P}}^{\mathcal{G}}\left(\gamma_{y}^{G}\right)(x)} .
$$

Now, by definition we have

$$
{ }^{*} \mathcal{R}_{\mathcal{L} \subset \mathcal{P}}^{\mathcal{G}}\left(\gamma_{y}^{G}\right)(x)=\left|C_{L}^{o}\left(x_{s}\right)^{F}\right|\left|C_{G}^{o}\left(x_{s}\right)^{F}\right|^{-1} \sum_{n \in C_{\mathcal{G}}\left(x_{s}\right)_{n i l}^{F}} \mathcal{Q}_{C_{\mathcal{L}}\left(x_{s}\right)}^{C_{\mathcal{G}}\left(x_{s}\right)}\left(n, x_{n}\right) \gamma_{y}^{G}\left(x_{s}+n\right) .
$$

Since by assumption $C_{G}^{o}\left(x_{s}\right) \subseteq L$, we have $C_{G}^{o}\left(x_{s}\right)=C_{L}^{o}\left(x_{s}\right)$, and so we get that

$$
{ }^{*} \mathcal{R}_{\mathcal{L} \subset \mathcal{P}}^{\mathcal{G}}\left(\gamma_{y}^{G}\right)(x)=\sum_{n \in C_{\mathcal{G}}\left(x_{s}\right)_{n i l}^{F}} \mathcal{Q}_{C_{\mathcal{G}}\left(x_{s}\right)}^{C_{\mathcal{G}}\left(x_{s}\right)}\left(n, x_{n}\right) \gamma_{y}^{G}\left(x_{s}+n\right)
$$

This formula shows that if $x_{s}$ is not $G^{F}$-conjugate to $y_{s}$, then ${ }^{*} \mathcal{R}_{\mathcal{L} \subset \mathcal{P}}^{\mathcal{G}}\left(\gamma_{y}^{G}\right)(x)=0$. Hence we may assume that $y_{s}=x_{s}$, and we have

$$
{ }^{*} \mathcal{R}_{\mathcal{L} \subset \mathcal{P}}^{\mathcal{G}}\left(\gamma_{y}^{G}\right)(x)=\left|C_{G}(y)^{F}\right| \sum_{n \in \mathcal{O}_{y_{n}}^{C_{G}\left(y_{s}\right)}} \mathcal{Q}_{C_{\mathcal{G}}\left(y_{s}\right)}^{C_{\mathcal{G}}\left(y_{s}\right)}\left(n, x_{n}\right)
$$

We now compute the quantity $\mathcal{Q}_{C_{\mathcal{G}}\left(y_{s}\right)}^{C_{\mathcal{G}}\left(y_{s}\right)}\left(n, x_{n}\right)$. By definition of Green functions, we have

$$
\mathcal{Q}_{C_{\mathcal{G}}\left(y_{s}\right)}^{C_{\mathcal{G}}\left(y_{s}\right)}\left(n, x_{n}\right)=\left|C_{G}^{o}\left(y_{s}\right)^{F}\right|^{-1} \operatorname{Trace}\left(\left(\phi(n), \phi\left(x_{n}\right)^{-1}\right) \mid H_{c}^{*}\left(C_{G}^{o}\left(y_{s}\right)^{F}\right)\right) .
$$

From [DM91, Proposition 10.8], we deduce that

$$
\begin{aligned}
\mathcal{Q}_{C_{\mathcal{G}}\left(y_{s}\right)}^{C_{\mathcal{G}}\left(y_{s}\right)}\left(n, x_{n}\right) & =\left|C_{G}^{o}\left(y_{s}\right)^{F}\right|^{-1} \operatorname{Trace}\left(\left(\phi(n), \phi\left(x_{n}\right)^{-1}\right) \mid \overline{\mathbf{Q}}_{\ell}\left[C_{G}^{o}\left(y_{s}\right)^{F}\right]\right) \\
& =\left|C_{G}^{o}\left(y_{s}\right)^{F}\right|^{-1} \sharp\left\{g \in C_{G}^{o}\left(y_{s}\right)^{F} \mid \phi(n) g \phi\left(x_{n}\right)^{-1}=g\right\} \\
& =\left|C_{G}^{o}\left(y_{s}\right)^{F}\right|^{-1} \sharp\left\{g \in C_{G}^{o}\left(y_{s}\right)^{F} \mid \operatorname{Ad}(g) x_{n}=n\right\} .
\end{aligned}
$$

From the last formula and (2), we deduce that ${ }^{*} \mathcal{R}_{\mathcal{L} \subset \mathcal{P}}^{\mathcal{G}}\left(\gamma_{y}^{G}\right)(x)=\left|C_{G}(y)^{F}\right|$ if $x$ is $G^{F}$ conjugate to $y$ and ${ }^{*} \mathcal{R}_{\mathcal{L} \subset \mathcal{P}}^{\mathcal{G}}\left(\gamma_{y}^{G}\right)(x)=0$ otherwise. From (1), it follows that $\mathcal{R}_{\mathcal{L} \subset \mathcal{P}}^{\mathcal{G}}\left(\gamma_{x}^{L}\right)=$ $\gamma_{x}^{G}$ 


\section{The Mackey formula and its applications}

In this section, we first discuss the validity of the Mackey formula for $\mathcal{R}_{\mathcal{L} \subset \mathcal{P}}^{\mathcal{G}}$; in the group case, this has been discussed by many authors including Deligne-Lusztig [DL83, Theorem 7], and Bonnafé [Bon98] [Bon00]. Here, we prove that the Mackey formula holds in the Lie algebra case whenever it does in the group case (assuming that $p$ is good for $G$ so that $\mathcal{R}_{\mathcal{L} \subset \mathcal{P}}^{\mathcal{G}}$ exists). To prove this, we follow [Bon98] where it is shown (in the group case) that the Mackey formula is equivalent to a formula on two-variable Green-functions. In a second part, we will see some consequences of the Mackey formula (well-known in the group case) such as the independence of $\mathcal{R}_{\mathcal{L} \subset \mathcal{P}}^{\mathcal{G}}$ from the parabolic subgroup $P$ or the commutation of $\mathcal{R}_{\mathcal{L} \subset \mathcal{P}}^{\mathcal{G}}$ with the duality map.

3.1. The Mackey formula: definition. The Mackey formula describes the composition ${ }^{*} \mathcal{R}_{\mathcal{L} \subset \mathcal{P}}^{\mathcal{G}} \circ \mathcal{R}_{\mathcal{M} \subset \mathcal{Q}}^{\mathcal{G}}: \mathcal{C}\left(\mathcal{M}^{F}\right) \rightarrow \mathcal{C}\left(\mathcal{L}^{F}\right)$. More precisely, the Mackey formula with respect to $(G, L, M, P, Q)$, is the following equality:

\subsection{1.}

$$
{ }^{*} \mathcal{R}_{\mathcal{L} \subset \mathcal{P}}^{\mathcal{G}} \circ \mathcal{R}_{\mathcal{M} \subset \mathcal{Q}}^{\mathcal{G}}=\sum_{x \in L^{F} \backslash \mathcal{S}_{G}(L, M)^{F} / M^{F}} \mathcal{R}_{\mathcal{L} \cap^{x} \mathcal{M} \subset \mathcal{L} \cap^{x} \mathcal{Q}^{\mathcal{L}}}{ }^{*} \mathcal{R}_{\mathcal{L}^{x} \cap^{x} \mathcal{M} \subset \mathcal{P} \cap^{x} \mathcal{M}}^{\circ} \operatorname{Ad}_{x}
$$

where $\mathcal{S}_{G}(L, M)$ denotes the set of $x \in G$ such that $L \cap{ }^{x} M$ contains a maximal torus of $G$.

3.2. The main result of this section. Here we state the Lie algebra analogue of a result of Bonnafé reducing the proof of the Mackey formula to the proof of a formula on two-variable Green functions so called the "Mackey formula for Green functions".

NotATion 3.2.1. If $H$ is a reductive group, we denote by $r k_{s s}(H)$ the semi-simple rank of $H$, i.e the rank of $H / Z_{H}^{o}$.

Let $L$ and $M$ be two $F$-stable Levi subgroups of $G$ and let $P$ and $Q$ be two parabolic subgroups of $G$ having respectively $L$ and $M$ as Levi subgroup. Then we denote by $\mathcal{T}(G, L, M)$ the set of triples $\left(G^{\prime}, L^{\prime}, M^{\prime}\right)$ such that:

(i) $G^{\prime}$ is an $F$-stable connected reductive subgroup of $G$ such that $G^{\prime}$ contains a maximal torus of $G$ and $r k_{s s}\left(G^{\prime}\right)<r k_{s s}(G)$,

(ii) $L^{\prime}$ and $M^{\prime}$ are two $F$-stable Levi subgroups of $G^{\prime}$ which are respectively $G^{F}$ conjugate to a subgroup of $L$ and a subgroup of $M$,

For each $\left(G^{\prime}, L^{\prime}, M^{\prime}\right) \in \mathcal{T}(G, L, M)$, we choose two parabolic subgroups $P^{\prime}$ and $Q^{\prime}$ of $G^{\prime}$ such that $L^{\prime}$ and $M^{\prime}$ are Levi factors respectively of $P^{\prime}$ and $Q^{\prime}$, and such that there exists $x, y \in G^{F}$ verifying $L^{\prime} \subset{ }^{x} L, M^{\prime} \subset{ }^{y} M$ and $P^{\prime} \subset{ }^{x} P, Q^{\prime} \subset{ }^{y} Q$.

Notation 3.2.2. If $\left(G^{\prime}, L^{\prime}, M^{\prime}\right) \in \mathcal{T}(G, L, M) \cup\{(G, L, M)\}$, we write $\left(G^{\prime}, L^{\prime}, M^{\prime}\right) \leq(G, L, M)$. This defines a partial order on $\mathcal{T}(G, L, M) \cup\{(G, L, M)\}$. 
Notation 3.2.3. If $\left(G^{\prime}, L^{\prime}, M^{\prime}\right) \leq(G, L, M)$, we denote by $\mathcal{G}^{\prime}, \mathcal{L}^{\prime}, \mathcal{M}^{\prime}, \mathcal{P}^{\prime}$ and $\mathcal{Q}^{\prime}$ the respective Lie algebras of $G^{\prime}, L^{\prime}, M^{\prime}, P^{\prime}$ and $Q^{\prime}$; with this notation, we write $\mathcal{R}_{\mathcal{L}^{\prime}}^{\mathcal{G}^{\prime}}$ instead of $\mathcal{R}_{\mathcal{L}^{\prime} \subset \mathcal{P}^{\prime}}^{\mathcal{G}^{\prime}}$

Notation 3.2.4. If $\left(G^{\prime}, L^{\prime}, M^{\prime}\right) \leq(G, L, M)$, we denote by $\mathcal{M}\left(G^{\prime}, L^{\prime}, M^{\prime}\right)$ the equality 3.1.1 (with $\left(G^{\prime}, L^{\prime}, M^{\prime}, P^{\prime}, Q^{\prime}\right)$ instead of $(G, L, M, P, Q)$ ) and by $M\left(G^{\prime}, L^{\prime}, M^{\prime}\right)$ the corresponding equality in the group case (see [Bon98]).

REMARK 3.2.5. The Mackey formula $\mathcal{M}(G, L, M)$ holds if and only if (*) $\forall f \in \mathcal{C}\left(\mathcal{L}^{F}\right), \forall g \in \mathcal{C}\left(\mathcal{M}^{F}\right)$, $\left(\mathcal{R}_{\mathcal{L}}^{\mathcal{G}}(f), \mathcal{R}_{\mathcal{M}}^{\mathcal{G}}(g)\right)_{\mathcal{G}^{F}}=\sum_{x \in L^{F} \backslash \mathcal{S}_{G}(L, M)^{F} / M^{F}}\left({ }^{*} \mathcal{R}_{\mathcal{L} \cap^{x} \mathcal{M}}^{\mathcal{L}}(f),{ }^{*} \mathcal{R}_{\mathcal{L}}^{x}{ }_{\mathcal{L} \cap} \mathcal{M}^{x} \circ \operatorname{Ad}_{x}(g)\right) \mathcal{L}^{F} \cap \operatorname{Ad}(x) \mathcal{M}^{F}$.

The next formula is somehow the analogue for Green functions of 3.2.5(*):

Definition 3.2.6 (The Mackey formula for Green functions). For $u \in \mathcal{G}^{F}$ and $v \in$ $\mathcal{L}^{F}$, we denote by $\mathcal{Q}_{\mathcal{L}}^{\mathcal{G}}(u,$.$) (resp. \mathcal{Q}_{\mathcal{L}}^{\mathcal{G}}(., v)$ ) the invariant function on $\mathcal{L}^{F}$ (resp. on $\mathcal{G}^{F}$ ) that takes the value 0 at non-nilpotent elements and that takes the value $\mathcal{Q}_{\mathcal{L}}^{\mathcal{G}}(u, v)$ at $v$ (resp. $u$ ). We call the Mackey formula for Green functions with respect to $(G, L, M)$ the following formula:

(*) $\forall u \in \mathcal{L}_{\text {nil }}^{F}, \forall v \in \mathcal{M}_{\text {nil }}^{F}$,

$$
\left.\left(\mathcal{Q}_{\mathcal{L}}^{\mathcal{G}}(., u), \mathcal{Q}_{\mathcal{M}}^{\mathcal{G}}(., v)\right)_{\mathcal{G}^{F}}=\sum_{x \in L^{F} \backslash \mathcal{S}_{G}(L, M)^{F} / M^{F}}\left(\mathcal{Q}_{\mathcal{L} \cap \mathcal{L}^{\mathcal{L}}}^{\mathcal{L}}(u, .), \mathcal{Q}_{\mathcal{L}{ }^{x} \mathcal{M}^{x}}{ }^{x} v, .\right)\right)_{\mathcal{L}^{F} \cap \operatorname{Ad}(x) \mathcal{M}^{F}} .
$$

Notation 3.2.7. We denote by $\mathcal{Q}(G, L, M)$ the formula 3.2.6(*) and by $Q(G, L, M)$ the corresponding formula in the group case [Bon98, 2.2].

REMARK 3.2.8. It is clear from our definition of the two-variable Green functions that the formula $\mathcal{Q}(G, L, M)$ holds exactly when $Q(G, L, M)$ does.

The following proposition is the main result of this section (see [Bon98, Proposition 2.3.6] for the group case).

PROPOSITION 3.2.9. The following assertions are equivalent,

(i) For any $\left(G^{\prime}, L^{\prime}, M^{\prime}\right) \leq(G, L, M)$, the Mackey formula for Green functions $\mathcal{Q}\left(G^{\prime}, L^{\prime}, M^{\prime}\right)$ holds.

(ii) For any $\left(G^{\prime}, L^{\prime}, M^{\prime}\right) \leq(G, L, M)$, the Mackey formula $\mathcal{M}\left(G^{\prime}, L^{\prime}, M^{\prime}\right)$ holds.

COROLlaRY 3.2.10. The following assertions are equivalent,

(i) For any $\left(G^{\prime}, L^{\prime}, M^{\prime}\right) \leq(G, L, M)$, the Mackey formula $\mathcal{M}\left(G^{\prime}, L^{\prime}, M^{\prime}\right)$ holds.

(ii) For any $\left(G^{\prime}, L^{\prime}, M^{\prime}\right) \leq(G, L, M)$, the Mackey formula $M\left(G^{\prime}, L^{\prime}, M^{\prime}\right)$ holds.

The corollary is a straightforward consequence of 3.2.9, 3.2.8 and the group version of 3.2.9 (which is [Bon98, Proposition 2.3.6]). 
3.3. Proof of 3.2.9. The proof of 3.2 .9 is entirely similar to that of its group version [Bon98]. We sketch it for the convenience of the reader.

For $f \in \mathcal{C}\left(\mathcal{L}^{F}\right)$ and $g \in \mathcal{C}\left(\mathcal{M}^{F}\right)$, define

$$
\begin{aligned}
\mathcal{R}_{\mathcal{L}, \mathcal{M}}^{\mathcal{G}}(f, g)= & \left(\mathcal{R}_{\mathcal{L}^{\mathcal{G}}}^{\mathcal{G}}(f), \mathcal{R}_{\mathcal{M}}^{\mathcal{G}}(g)\right)_{\mathcal{G}^{F}} \\
& -\sum_{x \in L^{F} \backslash \mathcal{S}_{G}(L, M)^{F} / M^{F}}\left({ }^{*} \mathcal{R}_{\mathcal{L} \cap^{x} \mathcal{M}}^{\mathcal{L}}(f),{ }^{*} \mathcal{R}_{\mathcal{L}^{x} \mathcal{M}^{x} \mathcal{M}} \circ \operatorname{Ad}_{x}(g)\right)_{\mathcal{L}^{F} \cap \operatorname{Ad}(x) \mathcal{M}^{F}},
\end{aligned}
$$

and for $u \in \mathcal{L}_{\text {nil }}^{F}$ and $v \in \mathcal{M}_{\text {nil }}^{F}$, define

$$
\begin{aligned}
\mathcal{Q}_{\mathcal{L}, \mathcal{M}}^{\mathcal{G}}(u, v)= & \left(\mathcal{Q}_{\mathcal{L}}^{\mathcal{G}}(., u), \mathcal{Q}_{\mathcal{M}}^{\mathcal{G}}(., v)\right)_{\mathcal{G}}{ }^{F} \\
& -\sum_{x \in L^{F} \backslash \mathcal{S}_{G}(L, M)^{F} / M^{F}}\left(\mathcal{Q}_{\mathcal{L} \cap^{x} \mathcal{M}}^{\mathcal{L}}(u, .), \mathcal{Q}_{\mathcal{L}^{x} \mathcal{M}^{x} \mathcal{M}}\left({ }^{x} v, .\right)\right)_{\mathcal{L}^{F} \cap \operatorname{Ad}(x) \mathcal{M}^{F}}
\end{aligned}
$$

The following result gives an expression of the $\mathcal{R}_{\mathcal{L}, \mathcal{M}}^{\mathcal{G}}$ in terms of $\mathcal{Q}_{\mathcal{L}, \mathcal{M}}^{\mathcal{G}}$, see [Bon98, Corollary 2.3.5] for the group case.

LEMMA 3.3.1. We assume that $\mathcal{M}\left(G^{\prime}, L^{\prime}, M^{\prime}\right)$ holds for all triples $\left(G^{\prime}, L^{\prime}, M^{\prime}\right)$ of $\mathcal{T}(G, L, M)$. Then for any $f \in \mathcal{C}\left(\mathcal{L}^{F}\right)$ and $g \in \mathcal{C}\left(\mathcal{M}^{F}\right)$ we have

$$
\mathcal{R}_{\mathcal{L}, \mathcal{M}}^{\mathcal{G}}(f, g)=\sum_{z \in z(\mathcal{G})^{F}} \sum_{v \in \mathcal{L}_{n i l}^{F}} \sum_{w \in \mathcal{M}_{n i l}^{F}} f(z+v) \overline{g(z+w)} \mathcal{Q}_{\mathcal{L}, \mathcal{M}}^{\mathcal{G}}(v, w) .
$$

The proof of [Bon98, Corollary 2.3.5] can be adapted without difficulties to the Lie algebra case.

Proof of 3.2.9. Assuming (i) and using 3.3.1, we can prove (ii) easily by induction on $\operatorname{dim} G^{\prime}+\operatorname{dim} L^{\prime}+\operatorname{dim} M^{\prime}$ where $\left(G^{\prime}, L^{\prime}, M^{\prime}\right)$ runs over the set of triples $\leq(G, L, M)$.

Assume that (ii) is true. Let $\left(G^{\prime}, L^{\prime}, M^{\prime}\right) \leq(G, L, M)$. We want to prove that for any $u \in \mathcal{L}^{\prime F}{ }_{n i l}^{F}$ and $v \in \mathcal{M}_{n i l}^{\prime F}$, we have $\mathcal{Q}_{\mathcal{L}^{\prime}, \mathcal{M}^{\prime}}^{\mathcal{G}^{\prime}}(u, v)=0$. Since the Mackey formula holds for any triple $\leq(G, L, M)$, it does for any triple $\leq\left(G^{\prime}, L^{\prime}, M^{\prime}\right)$ and so by 3.3.1, for any $u \in \mathcal{L}^{\prime}{ }_{n i l}$. and $v \in \mathcal{M}_{\text {nil }}^{\prime F}$, we get that (see notation 1.0.4):

$$
\mathcal{R}_{\mathcal{L}^{\prime}, \mathcal{M}^{\prime}}^{\mathcal{G}^{\prime}}\left(\gamma_{u}^{L^{\prime}}, \gamma_{v}^{M^{\prime}}\right)=\left|L^{\prime F}\right|\left|M^{\prime F}\right| \mathcal{Q}_{\mathcal{L}^{\prime}, \mathcal{M}^{\prime}}^{\mathcal{G}^{\prime}}(u, v)
$$

But by assumption, the left hand side of the above equation is equal to 0 , so $\mathcal{Q}_{\mathcal{L}^{\prime}, \mathcal{M}^{\prime}}^{\mathcal{G}^{\prime}}(u, v)=$ 0 .

3.4. Consequences. By [Bon98], there exists an integer $q_{o}$, depending only on $G$, such that if $q>q_{o}$, then for any $F$-stable Levi subgroups $L$ and $M$ of $G$, the Mackey formula $M\left(G^{\prime}, L^{\prime}, M^{\prime}\right)$ holds for any triple $\left(G^{\prime}, L^{\prime}, M^{\prime}\right) \leq(G, L, M)$. Hence by 3.2.10 we have:

THEOREM 3.4.1. If $q>q_{o}$, the Mackey formula $\mathcal{M}(G, L, M)$ holds for any $F$-stable Levi subgroups $L$ and $M$. 
REMARK 3.4.2. In some cases, we can prove that the Mackey formula $\mathcal{M}(G, L, M)$ holds without assumption on $q$. This is the case for instance if $G$ is of type $A_{n}$, or if $L$ or $M$ is a maximal torus. These results follow from their group versions (see [Bon00] if $G$ is of type $A_{n}$, and see [DL83] if $L$ or $M$ is a maximal torus) together with 3.2.10.

Proposition 3.4.3. If $q>q_{o}$, the Deligne-Lusztig induction $\mathcal{R}_{\mathcal{L} \subset \mathcal{P}}^{\mathcal{G}}$ does not depend on the choice of the parabolic subgroup $P$ of $G$ having L as a Levi subgroup.

PROOF. The proof is entirely similar to that of [DM91, Proposition 6.8].

NotAtion 3.4.4. We denote $\mathcal{R}_{\mathcal{L}}^{\mathcal{G}}$ instead of $\mathcal{R}_{\mathcal{L} \subset \mathcal{P}}^{\mathcal{G}}$; this is justified in view of 3.4.3.

Now we are interested in the relationship between duality maps and Deligne-Lusztig induction. This relationship is known in the group case, see [DM91, p.66]; the corresponding formula for Lie algebras is given in the following theorem:

THEOREM 3.4.5. Assume $q>q_{\text {o }}$. Let L be an F-stable Levi subgroup of $G$ and let $\mathcal{L}$ be its Lie algebra. Then

$$
\mathcal{D}_{\mathcal{G}} \circ \mathcal{R}_{\mathcal{L}}^{\mathcal{G}}=\varepsilon_{G} \varepsilon_{L} \mathcal{R}_{\mathcal{L}}^{\mathcal{G}} \circ \mathcal{D}_{\mathcal{L}}
$$

where $\varepsilon_{G}=(-1)^{\mathbf{F}_{q}-\operatorname{rank}(G)}$.

PROOF. The proof is entirely similar to that of [DM91, Theorem 8.11] since the only properties of $\mathcal{R}_{\mathcal{L}}^{\mathcal{G}}$ it uses are transitivity (see 2.3.6), the Mackey formula for $(G, M, L)$ with $M$ a $G$-split Levi subgroup of $G$ and the following formula (which is easy to verify)

$$
\operatorname{Ad}_{x-1} \circ \mathcal{R}_{x}^{x} \mathcal{L} \circ \operatorname{Ad}_{x}=\mathcal{R}_{\mathcal{M}}^{\mathcal{L}}
$$

for any $x \in G^{F}$ and any $\mathcal{L}, \mathcal{M}$ such that $\mathcal{M} \subset \mathcal{L}$.

\section{References}

[Bon98] C. BonnAFÉ, Formule de Mackey pour q grand, J. Algebra 201 (1998), no. 1, $207-232$.

[Bon00] C. BonnafÉ, Mackey formula in type A, Proc. London Math. Soc. (3) 80 (2000), no. 3, 545-574.

[Bon02] C. BonNAFÉ, A note on centralizers of unipotent elements, preprint of Laboratoire de mathématiques de Besancon 19 (2002).

[Bor] A. BOREL, Linear algebraic groups, Springer-Verlag (second enlarged edition).

[De177] P. Deligne, Cohomologie étale, Springer-Verlag, Berlin, 1977, Séminaire de Géométrie Algébrique du Bois-Marie SGA $4 \frac{1}{2}$, Avec la collaboration de J. F. Boutot, A. Grothendieck, L. Illusie et J. L. Verdier, Lecture Notes in Mathematics, Vol. 569.

[DL76] P. Deligne and G. LuszTig, Representations of reductive groups over finite fields, Ann. of Math. (2) 103 (1976), no. 1, 103-161.

[DL83] P. Deligne and G. LuszTIG, Duality for representations of a reductive group over a finite field. II, J. Algebra 81 (1983), no. 2, 540-545.

[DM87] FranÇOIS DIGNE and JEAN MiChel, Foncteurs de Lusztig et caractères des groupes linéaires et unitaires sur un corps fini, J. Algebra 107 (1987), no. 1, 217-255. 
[DM91] FranÇOIS Digne and Jean Michel, Representations of finite groups of Lie type, Cambridge University Press, Cambridge, 1991.

[Gre55] J. A. GreEn, The characters of the finite general linear groups, Trans. Amer. Math. Soc. 80 (1955), 402-447.

[Kaw82] N. KAWANAKA, Fourier transforms of nilpotently supported invariant functions on a simple Lie algebra over a finite field, Invent. Math. 69 (1982), no. 3, 411-435.

[Kaz77] D. KaZHDan, Proof of Springer's hypothesis, Israel J. Math. 28 (1977), no. 4, 272-286.

[Leh96] Gus LeHRER, The space of invariant functions on a finite Lie algebra, Trans. Amer. Math. Soc. 348 (1996), no. 1, 31-50.

[Lus76] G. LuszTig, On the Finiteness of the Number of Unipotent Classes, Invent. Math. 34 (1976), 201-213.

[Lus86] G. LuszTig, On the Character Values of Finite Chevalley Groups at Unipotent Elements, J. Algebra 104 (1986), no. 2, 146-194.

[Slo80] Peter SLOdowy, Simple singularities and simple algebraic groups, Springer, Berlin (1980).

[Spr69] T. A. SPRINGER, The unipotent variety of a semi-simple group, Algebraic Geometry (Internat. Colloq., Tata Inst. Fund. Res., Bombay, 1968), Oxford Univ. Press, London (1969), 373-391.

[Spr76] T. A. SpRINGER, Trigonometric sums, Green functions of finite groups and representations of Weyl groups, Invent. Math. 36 (1976), 173-207.

Present Address:

Department of Mathematics, Sophia University,

KiOICHO, CHIYODA-KU, TOKYO 102-8554, JAPAN. 